\title{
Lois analytiques pour les écoulements en cavité rotor-stator
}

\author{
Sébastien Poncet ${ }^{1, a}$, Marie-Pierre Chauve ${ }^{2}$ et Patrice Le Gal ${ }^{2}$ \\ 1 Laboratoire de Modélisation et Simulation Numérique en Mécanique - Génie des Procédés, UMR 6181 CNRS, \\ Universités d'Aix-Marseille I, II \& III, IMT La Jetée, Technopôle Château-Gombert, 38 rue F. Joliot-Curie, \\ 13451 Marseille Cedex 20, France \\ 2 Institut de Recherche sur les Phénomènes Hors Équilibre, UMR 6594 CNRS, Universités d'Aix-Marseille I \& II, \\ Technopôle Château-Gombert, 49 rue F. Joliot-Curie, BP 146, 13384 Marseille Cedex 13, France
}

Reçu le 3 avril 2007, accepté le 9 janvier 2008

\begin{abstract}
Résumé - Cette étude concerne les écoulements de type rotor-stator à couches limites séparées soumis à un flux axial. Suivant l'analyse faite par Schlichting [1] dans le cas d'un disque tournant de rayon infini, on détermine des lois analytiques simples permettant de prévoir le coefficient d'entraînement $K$ du fluide à partir des paramètres de contrôle (taux de rotation, flux, espace interdisque, taux de prérotation) pour divers régimes d'écoulement (laminaire ou turbulent) et différentes configurations (géométrie, rotor lisse, rugueux ou aileté). Cette approche analytique est validée par d'extensives mesures de vitesse et de pression.
\end{abstract}

Mots clés : Cavité rotor-stator / modèle analytique / LDA / mesures de pression

Abstract - Analytical laws for rotor-stator flows. The evolution of the entrainment coefficient $K$ of the rotating fluid in a rotor-stator cavity with an axial throughflow is studied according to the flow parameters. Following the analysis performed by Schlichting [1] in the single disk case, we determine analytical laws for the prediction of $K$, which depends in each case on a different local flow rate coefficient. The influence of all parameters (geometry of the cavity, rotation rate, throughflow, prerotation level, rugosity, blades) are investigated. The behavior of the entrainement coefficient is confirmed by extensive measurements realized in water for a Batchelor type of flow with separated boundary layers by the means of a laser Doppler anemometer. The results are also compared to those performed by pressure transducers.

Key words: Rotor-stator cavity / analytical model / LDA / pressure measurements

\section{Introduction}

Les écoulements confinés entre un disque tournant (le rotor) et un disque fixe (le stator) présentent un intérêt industriel certain dans des domaines comme l'informatique, la chimie et principalement dans celui des turbomachines. La cavité interdisque considérée dans cette étude représente une partie simplifiée de la turbopompe à hydrogène liquide du moteur Vulcain (SNECMA Moteurs). Les écoulements interdisques dans cette turbopompe sont responsables pour $90 \%$ des efforts axiaux sur les rotors dus aux surpressions de l'hydrogène liquide. Pour un bon fonctionnement, il faut rendre la résultante totale de ces efforts nulle et ce quel que soit le régime d'écoulement. Différents types d'équilibrage axial du rotor peuvent être mis en place afin de minimiser ces efforts. Ces dispositifs contrôlent les efforts à travers le gradient de pression radial et l'effet centrifuge. Il est donc capital de prévoir

a Auteur pour correspondance : poncet@l3m.univ-mrs.fr au mieux les distributions radiales de pression dans ces écoulements en fonction des paramètres de contrôle : le taux de rotation du rotor $\Omega$, le flux imposé $Q$ et l'espace interdisque $h$.

Les écoulements en cavité rotor-stator fermée ou partiellement fermée, se classent en deux grandes familles selon la taille de l'espace interdisque $h$. Pour chaque famille, l'écoulement pouvant être laminaire ou turbulent, Daily et Nece [2] déterminent quatre régimes d'écoulement grâce à deux paramètres adimensionnels : le nombre de Reynolds $R e=\Omega R_{2}^{2} / \nu$ ( $\nu$ la viscosité cinématique du fluide) basé sur le rayon du disque tournant $R_{2}$ et le rapport de forme de la cavité $G=h / R_{2}$. Lorsque $h$ est assez grand, les couches de Bödewadt liée au stator et d'Ekman liée au rotor sont séparées par un coeur en rotation et la structuration de l'écoulement est dite de Batchelor. Pour $h$ assez faible, elles sont jointes et l'écoulement est de type «Couette de torsion». Les principales expériences sur les écoulements de Batchelor 


\section{Nomenclature}

\begin{tabular}{|c|c|}
\hline$c$ & épaulement du cylindre extérieur, m \\
\hline$C_{\mathrm{f}}$ & coefficient de frottement \\
\hline$c_{i}$ & espace radial entre le rotor \\
\hline & et le cylindre extérieur, m \\
\hline$C_{\mathrm{p}}$ & coefficient de pression \\
\hline$C_{\mathrm{q}_{\mathrm{lam}}}, C_{\mathrm{qr}_{\mathrm{r}}}, C_{\mathrm{q}_{\mathrm{rug}}}$ & coefficients locaux de débit \\
\hline$C_{\mathrm{w}}$ & coefficient global de débit \\
\hline$G$ & rapport d'aspect de la cavité \\
\hline$h$ & espace interdisque, $\mathrm{m}$ \\
\hline$h_{i}$ & espace axial entre \\
\hline & le rotor et l'épaulement, m \\
\hline$K$ & coefficient d'entraînement du fluide \\
\hline$k_{\mathrm{s}}$ & hauteur moyenne des rugosités, m \\
\hline$K_{\mathrm{v}}$ & taux de prérotation \\
\hline$P$ & pression, bars \\
\hline$Q$ & flux axial, $\mathrm{m}^{3} \cdot \mathrm{s}^{-1}$ \\
\hline$r, \theta, z$ & $\begin{array}{l}\text { coordonnées radiale, tangentielle } \\
\text { et axiale, } \mathrm{m}\end{array}$ \\
\hline$R_{1}, R_{2}, R_{3}, R_{4}$ & $\begin{array}{l}\text { rayons caractéristiques } \\
\text { de la cavité, m }\end{array}$ \\
\hline$R_{\mathrm{c}}$ & paramètre de courbure de la cavité \\
\hline$R e$ & nombre de Reynolds de rotation \\
\hline$V_{r}, V_{\theta}, V_{z}$ & $\begin{array}{l}\text { composantes radiale, tangentielle } \\
\text { et axiale de la vitesse, } \mathrm{m} \cdot \mathrm{s}^{-1}\end{array}$ \\
\hline$\delta$ & $\begin{array}{l}\text { recouvrement du rotor } \\
\text { sur l'épaulement, m }\end{array}$ \\
\hline$\delta_{\mathrm{E}}$ & $\begin{array}{l}\text { épaisseur de la couche } \\
\text { limite d'Ekman, m }\end{array}$ \\
\hline$\nu$ & viscosité cinématique du fluide, $\mathrm{m}^{2} \cdot \mathrm{s}^{-1}$ \\
\hline$\Omega$ & $\begin{array}{l}\text { taux de rotation du disque } \\
\text { tournant, } \mathrm{rad} . \mathrm{s}^{-1}\end{array}$ \\
\hline$\rho$ & masse volumique du fluide, $\mathrm{kg} \cdot \mathrm{m}^{-3}$ \\
\hline * & désigne une quantité adimensionnée \\
\hline
\end{tabular}

en cavité fermée sont celles d'Itoh et al. [3] et de Cheah et al. [4] qui ont fourni des données de référence pour les champs moyen et turbulent respectivement pour $G=0,08$ et $G=0,127$. Les écoulements en cavité fermée sont devenus ces dernières années des écoulements tests pour les simulations numériques directes (DNS) et les méthodes LES (Large Eddy Simulation). Cependant, ces approches sont, pour l'instant, limitées par la puissance de calculs à l'étude d'écoulements faiblement turbulents et la convergence statistique de la solution demande de trop nombreuses heures de calculs pour que ces approches soient utilisées dans l'industrie.

On considère dans la suite les écoulements à couches limites séparées lorsqu'un flux axial est imposé. Dans les systèmes rotor-stator soumis à un flux, on observe toujours quatre régimes d'écoulement mais les seuils sont différents du fait de l'introduction d'un nouveau paramètre de débit. Jimbo [5] a étudié l'effet d'un flux centripète sur un écoulement turbulent en cavité rotorstator. Il n'a pas noté d'influence de l'espace interdisque $h$ sur le gradient de pression radial. Kurokawa et Toyokura [6] ont proposé un modèle à une dimension pour calculer le coefficient d'entraînement du fluide $K$ défini comme le rapport entre la vitesse tangentielle du fluide dans le cour et celle du disque au même rayon. Ils ont montré l'influence dominante du flux centripète sur la détermination de $K$ et sur la distribution radiale de la pression. Ceci a été confirmé par les mesures de vitesse et de pression et par le modèle analytique de Debuchy et al. [6]. Nous verrons dans la section 3 que $K$ est directement lié au gradient de pression radial. Ainsi, $K$ est une quantité pertinente pour calculer les efforts axiaux sur le rotor.

La principale étude concernant les écoulements rotorstator soumis à un flux centrifuge est celle de Daily et al. [7]. Ils ont montré que la géométrie d'entrée affecte sensiblement la vitesse moyenne dans la cavité mais pas la distribution de pression. L'écoulement garde la structuration de Batchelor jusqu'à une certaine valeur du flux à partir de laquelle l'écoulement devient de type Stewartson et $K$ tend alors vers 0 . Aucun effet du rapport de forme de la cavité $G$ n'a été observé sur $K$. Récemment, Poncet et al. [8] ont comparé des mesures de vitesse aux prévisions du modèle RSM (Reynolds Stress Modeling) d'Elena et Schiestel [9]. Ils ont caractérisé en particulier la transition entre les structurations de Batchelor et de Stewartson. Cette transition est continue, indépendante du rapport d'aspect de la cavité et peut être caractérisée par un nombre de Rossby basé sur l'espace radial entre le rotor et le cylindre extérieur. Le lecteur peut se référer à l'ouvrage d'Owen et Rogers [10] et à la thèse de Poncet [11] pour une revue exhaustive des études concernant les écoulements de type rotor-stator avec ou sans flux.

L'objectif de cette étude est de déterminer des relations simples entre le taux de rotation du fluide $K$ et les paramètres de contrôle pour différents régimes d'écoulement et différentes géométries. L'approche analytique est complétée par des mesures extensives de vitesse et de pression.

\section{Dispositif expérimental}

\subsection{Cavité rotor-stator}

La cavité correspond à deux disques lisses (Fig. 1a), l'un en rotation et l'autre fixe, bornés par deux cylindres coaxiaux de hauteur $h$ variable entre 3 et $12 \mathrm{~mm}$. Le rayon extérieur de la cavité est $R_{3}+c$ avec $R_{3}=253 \mathrm{~mm}$ et $c$ la profondeur de l'épaulement, qui peut varier entre 0 et $5 \mathrm{~mm}$. Le rotor (rayon $R_{2}=250 \mathrm{~mm}$ ) et le moyeu central (rayon $R_{1}=38,5 \mathrm{~mm}$ ) qui lui est attaché sont entraînés par un moteur asynchrone triphasé d'une puissance de $5,5 \mathrm{~kW}$ permettant d'atteindre une vitesse de rotation $\Omega=600$ tr.min ${ }^{-1}$ à $1 \%$ près. Un flux $Q$ centrifuge $(Q<0)$ ou centripète $(Q>0)$ peut être imposé grâce à une pompe Wilo (IP-E50/5-28) centrifuge monocellulaire 


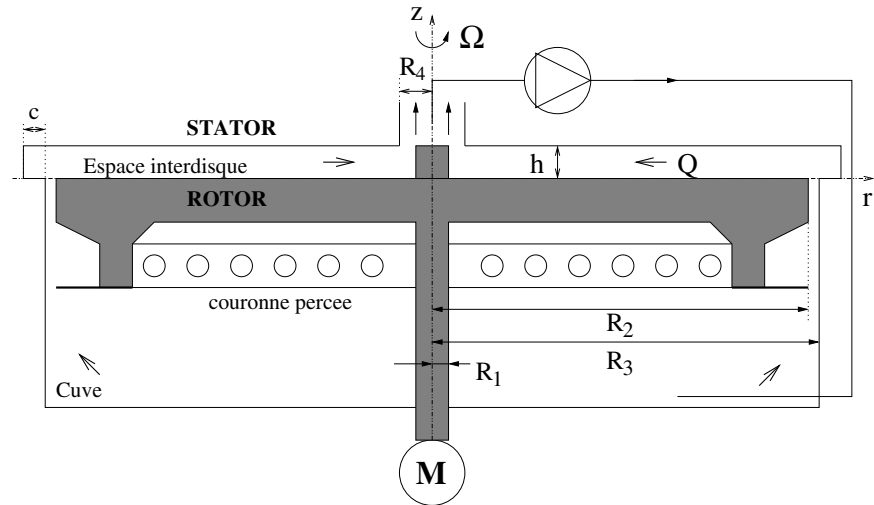

(a)
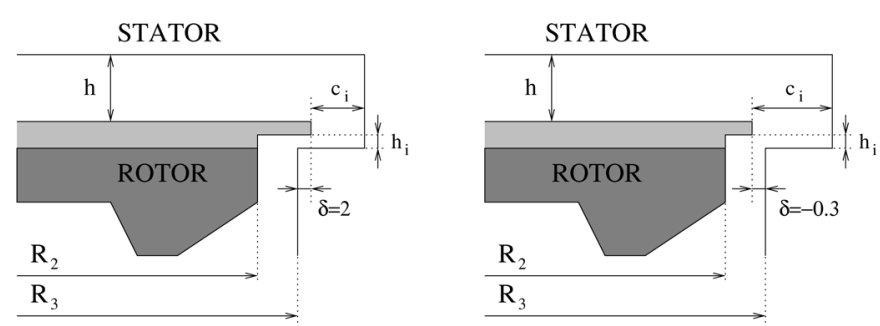

(b)

Fig. 1. (a) Schéma général du dispositif interdisque avec $R_{1}=$ $38,5, R_{2}=250, R_{3}=253, R_{4}=55,1 \leq h \leq 12$ et $0 \leq c \leq$ 5 ; (b) Géométries considérées en périphérie de la cavité avec $c_{i}=3, \delta=[-0,3 ; 2]$ et $h_{i}$ variable. Toutes les longueurs sont données en $\mathrm{mm}$.

basse pression qui permet d'assurer un débit d'au moins $4,51 . \mathrm{s}^{-1}$ avec une erreur sur la valeur du débit de l'ordre de $1 \%$.

La géométrie en périphérie de la cavité peut être modifiée pour étudier l'influence d'ajutages hauts sur l'écoulement de base. On dispose de 12 disques pouvant être superposés successivement au rotor et de 2 viroles (stator + cylindre extérieur). Selon les valeurs de $\delta=[-0,3,2] \mathrm{mm}$, le recouvrement en entrée est négatif ou positif (Fig. 1b). Pour la valeur de $\delta$ positive, on dispose de 6 disques de rayon $255 \mathrm{~mm}$ superposés successivement au disque tournant pour disposer de 6 hauteurs $h_{i}=[0,34 ; 0,45 ; 0,58 ; 0,85 ; 0,97 ; 1,09] \mathrm{mm}$ $( \pm 0,05 \mathrm{~mm})$. Pour la valeur de $\delta$ négative, on dispose de 6 disques de rayon $252,7 \mathrm{~mm}$ et donc de 6 hauteurs $h_{i}=[0,3 ; 0,45 ; 0,6 ; 0,78 ; 0,94 ; 1,15] \mathrm{mm}$. On peut donc étudier 12 configurations d'écoulement en entrée en dehors du cas de base. Les deux viroles ont un épaulement différent de sorte que, dans toutes ces configurations, l'espace radial $c_{i}$ est maintenu à une valeur constante de $3 \mathrm{~mm}$.

Dans le cas d'un flux centripète, le fluide est entraîné en prérotation par une couronne percée liée au rotor. Le coefficient de prérotation $K_{\mathrm{v}}$ est défini comme le rapport entre la vitesse tangentielle $V_{\theta}$ du fluide dans l'espace radial entre le rotor et l'épaulement $\left(r_{\mathrm{e}}=\left(R_{2}+R_{3}\right) / 2\right)$ et celle qu'aurait le disque au même rayon $\Omega r_{\mathrm{e}}$. Des mesures de vitesse par Poncet [11] ont montré que $0,5 \leq K_{\mathrm{v}} \leq 0,6$ suivant le débit centripète imposé. L'objectif de ce dispositif est d'avoir une valeur de $K_{\mathrm{v}}$ proche de celle rencontrée dans les turbopompes.

Un système de refroidissement a été installé sur le dispositif afin de maintenir la température de l'eau constante : $T=23 \pm 0,5{ }^{\circ} \mathrm{C}$ (viscosité cinématique $\nu=0,946 \pm 0,011 \mathrm{cSt}$ ). Il est à noter que le système est maintenu, au repos, à une pression constante de 2 bars pour éviter les effets de cavitation.

\subsection{Paramètres de l'écoulement}

L'écoulement hydrodynamique dépend principalement de quatres paramètres de contrôle : le rapport d'aspect $G$, le paramètre de courbure $R_{\mathrm{c}}$, le nombre de Reynolds $R e$ basé sur le rayon extérieur du disque tournant et un coefficient de débit volumique $C_{\mathrm{w}}$ définis par :

$$
\begin{gathered}
0,012 \leq G=\frac{h}{R_{2}} \leq 0,048 \quad R_{\mathrm{c}}=\frac{R_{2}+R_{1}}{R_{2}-R_{1}}=1,36 \\
R e=\frac{\Omega R_{2}^{2}}{\nu} \leq 4,15 \times 10^{6} \\
-2,6 \times 10^{4} \leq C_{\mathrm{w}}=\frac{Q}{\nu R_{2}} \leq 3,1 \times 10^{4}
\end{gathered}
$$

\subsection{Dispositif de mesures}

Les mesures de vitesse se font par un anémomètre laser Doppler (LDA) à deux composantes (Dantec Dynamics) placé au-dessus du stator. Cette technique non intrusive donne accès aux composantes radiale $V_{r}$ et orthoradiale $V_{\theta}$ de la vitesse et aux trois composantes associées du tenseur de Reynolds (non présentées ici) dans le plan vertical $(r, z)$ à un azimut donné. Les particules « Optimage PIV Seeding Powder $»$, choisies comme traceurs, sont des particules anisotropes, réflectives, à sédimentation lente dont la taille $30 \mu \mathrm{m}$ est supérieure à l'échelle du mouvement brownien $(5 \mu \mathrm{m})$ et inférieure à l'échelle de Kolmogorov (50 $\mu \mathrm{m}$ au plus haut nombre de Reynolds considéré). Les mesures sont rendues plus délicates dans la couche limite du rotor à cause de l'agglomération de particules qui sédimentent et donc à la difficulté d'ensemencer correctement l'écoulement. Il est à noter que la taille du volume de mesure selon la direction axiale $z(0,8 \mathrm{~mm})$ n'est pas négligeable dans certains cas, comparée à l'épaisseur des couches limites et à l'espace interdisque $h$. Nos mesures montrent que 1000 points sont suffisants pour une bonne convergence statistique des vitesses moyennes [11] avec une précision sur la mesure des vitesses moyennes de l'ordre de $2 \%$.

Les mesures de pression sont effectuées à l'aide de six capteurs de la série $33 S$ de la société Keller. Ce sont des transmetteurs de pression piézorésistifs de haute précision $\left(0,05 \%\right.$ entre 10 et $\left.40{ }^{\circ} \mathrm{C}\right)$, qui supportent une pression maximale de 20 bars. L'affichage des données est à 1 mbar près. On dispose donc de six stations de mesures de pression disposées radialement sur le stator en 
$r=[93,110,140,170,200,230] \mathrm{mm}$. Des mesures de pression par capteurs embarqués [12] ont montré que la pression sur le rotor et celle sur le stator sont en fait les mêmes à $2,5 \%$ près (conséquence du théorème de TaylorProudman). Les prédictions du modèle RSM de Poncet et al. $[8,11,13]$ ont confirmé le fait qu'il n'y a pas de gradient axial de pression. Ceci justifie le choix de placer les capteurs sur le stator.

\section{3 Écoulement de base}

On définit les grandeurs adimensionnées suivantes : $V_{\theta}^{*}=V_{\theta} /(\Omega r), V_{r}^{*}=V_{r} /(\Omega r), r^{*}=r / R_{2}, z^{*}=z / h$, de sorte que $z^{*}=0$ sur le rotor et $z^{*}=1$ sur le stator. Les résultats sur l'écoulement de base présentés figure 2 concernent les écoulements turbulents à couches limites séparées (régime IV de [2]).

L'écoulement de base en cavité fermée (Fig. 2b) est de type Batchelor à couches limites séparées par un coeur en rotation quasi solide $\left(V_{\theta}^{*}\left(z^{*}=0,5\right)=K=0,432\right.$, $\left.V_{r}^{*} \simeq 0\right)$. La couche d'Ekman sur le rotor est centrifuge, alors que celle de Bödewadt liée au stator est centripète. Les profils axiaux de la composante axiale de la vitesse $V_{z}^{*}$ ne sont pas représentés car elle vaut pratiquement zéro dans toute la cavité et ce quelles que soient les valeurs des paramètres de contrôle. Si on impose un flux axial centripète (Fig. 2a), l'écoulement conserve la structuration de type Batchelor avec une valeur du coefficient d'entraînement $K$ plus élevée. Le cœur peut alors tourner plus vite que le rotor : $K>1$. L'écoulement radial est essentiellement confiné le long du stator. À l'inverse, lorsqu'on impose un flux centrifuge, la structuration est de type Stewartson avec une seule couche limite sur le rotor. $K$ tend alors vers zéro et l'écoulement radial est purement centrifuge. La transition entre ces deux types de structuration est étudiée en détails dans [8].

Des mesures de pression ont également été faites à l'aide de 6 capteurs de pression placés sur le stator selon la direction radiale. On choisit de prendre, comme pression de référence, la pression $P$ mesurée à la position radiale extérieure $r^{*}=0,92$. On définit ainsi le coefficient de pression suivant : $C_{\mathrm{p}}=P^{*}\left(r^{*}\right)-P^{*}(0,92)$. La pression adimensionnée est donnée par : $P^{*}=2 P /\left(\rho \Omega^{2} R_{2}^{2}\right)$ où $\rho$ est la masse volumique de l'eau. Il est possible de calculer le coefficient $K$ à partir du gradient de pression radial. Nous avons vu précédemment, que pour les écoulements de Batchelor, le cœur en rotation est caractérisé par $V_{r} \simeq$ $V_{z} \simeq 0$ et $V_{\theta}$ constante. Ainsi, l'équation de Navier-Stokes pour la composante tangentielle se réduit donc, dans le coeur, à l'équilibre entre la force centrifuge et le gradient de pression radial :

$$
\rho V_{\theta}^{2} / r=\mathrm{d} P / \mathrm{d} r
$$

Sous la forme adimensionnée, on obtient finalement une équation liant le gradient de pression radial au coefficient d'entraînement $K$ :

$$
\mathrm{d} C_{\mathrm{p}}\left(r^{*}\right) / \mathrm{d} r^{*}=\mathrm{d} P^{*} / \mathrm{d} r^{*}=2 K^{2} r^{*}
$$

(a)
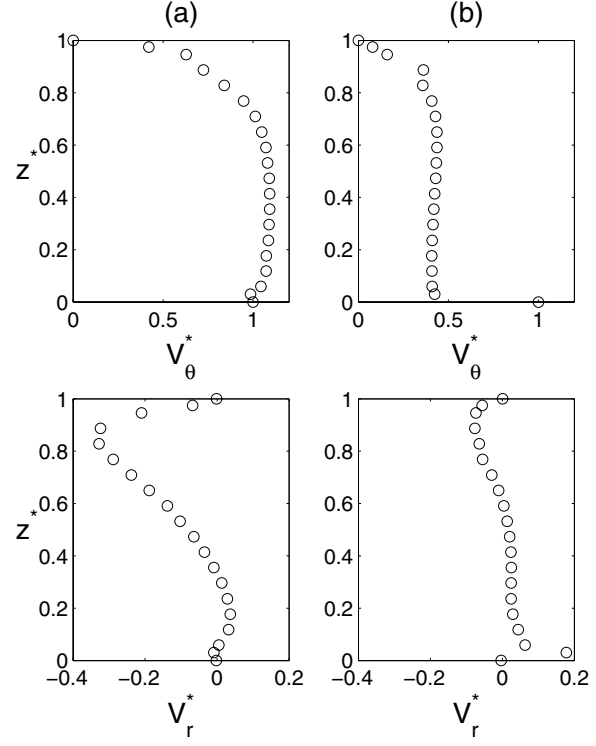

(c)
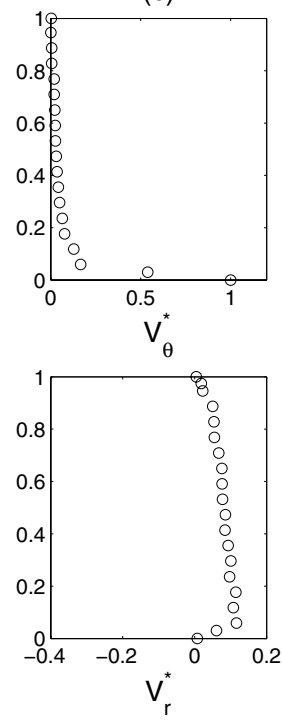

Fig. 2. Profils axiaux des composantes radiale $V_{r}^{*}$ et tangentielle $V_{\theta}^{*}$ de la vitesse moyenne à $r^{*}=0,56$ pour $R e=$ $1,04 \times 10^{6}, G=0,036$ et trois valeurs de $C_{\mathrm{w}}$ : (a) $C_{\mathrm{w}}=5929$, (b) $C_{\mathrm{w}}=0$, (c) $C_{\mathrm{w}}=-5929$.

La figure 3 a présente les variations du coefficient $C_{\mathrm{p}}$ en fonction de la position radiale $r^{*}$ pour quelques cas significatifs. Logiquement, la pression décroît de la périphérie vers le centre de la cavité : $C_{\mathrm{p}}$ est donc toujours négatif. À un rayon et un nombre de Reynolds donnés, $C_{\mathrm{p}}$ décroît pour des valeurs croissantes du coefficient de débit $C_{\mathrm{w}}$. A contrario, pour une valeur donnée de $C_{\mathrm{w}}, C_{\mathrm{p}}$ croît pour des valeurs croissantes de $R e$. Ces résultats sont en accord avec les mesures de Debuchy et al. [14].

Selon la relation (2), on peut déterminer le coefficient d'entraînement $K$ à partir de $C_{\mathrm{p}}$. On calcule donc la dérivée par morceaux de $C_{\mathrm{p}}$ par différences finies en choisissant le degré du polynôme le mieux adapté pour obtenir la valeur de $K$. Ainsi sur la figure $3 \mathrm{~b}$, on compare les variations de $K$ selon la position radiale $r^{*}$ pour des données obtenues par mesures de pression et par LDA. Les résultats sont en excellent accord. Les légères différences viennent du calcul de la dérivée de $C_{\mathrm{p}}$ à partir de seulement six points et de l'hypothèse de vitesse radiale nulle, qui est moins pertinente pour des forts flux centripètes. Si on est capable de prévoir la valeur du coefficient $K$ à partir de $\Omega, h, Q, r$ et $K_{\mathrm{v}}$, on peut en déduire le coefficient de pression $C_{\mathrm{p}}$ et ainsi déterminer les efforts axiaux sur le rotor.

\section{Modèle analytique pour les écoulements turbulents entre deux disques lisses}

L'objectif est de déterminer une relation liant le coefficient d'entraînement du fluide $K$ et les différents paramètres de l'écoulement [15]. On considère dans cette section un écoulement turbulent à couches limites séparées confiné entre un disque tournant et un disque 


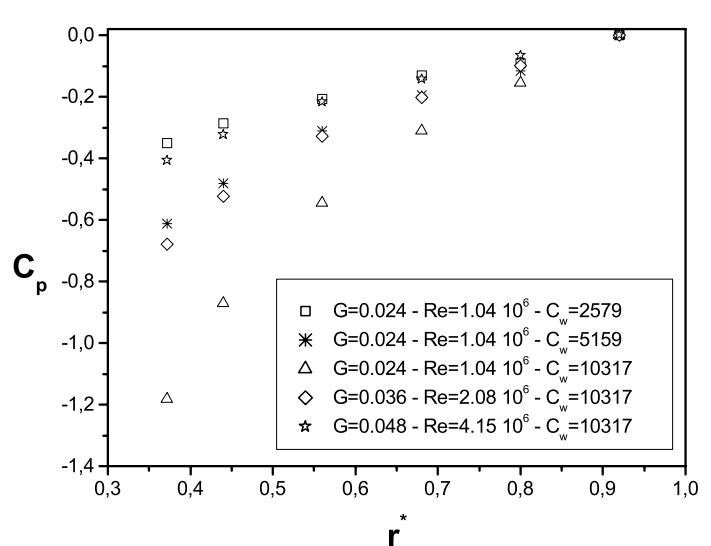

(a)

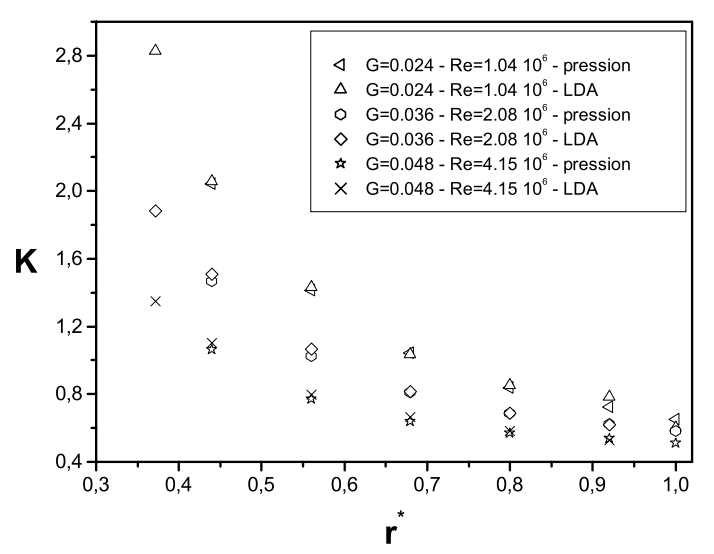

(b)

Fig. 3. (a) Distributions radiales du coefficient de pression $C_{\mathrm{p}}$ pour diverses conditions d'écoulement, (b) Comparaison entre les valeurs de $K$ obtenues à partir des mesures de pression et celles obtenues à partir des mesures de vitesse pour $C_{\mathrm{w}}=$ 10317 .

fixe tous deux hydrodynamiquement lisses. Reprenant l'analyse de Schlichting [1] dans le cas d'un disque tournant de rayon infini, on suppose que les profils de vitesse dans la couche d'Ekman évoluent selon une loi de puissance classique en $1 / 7$. Ainsi, le coefficient de frottement $C_{\mathrm{f}}$, égal par définition à la tension de cisaillement normalisée $\tau_{0}$, peut être donné par la formule de Dean : $C_{\mathrm{f}_{\text {moy }}}=0,073 R e_{\text {moy }}^{-1 / 4}$, où $R e_{\text {moy }}$ est le nombre de Reynolds basé sur $U_{\text {moy }}=(K+1) \Omega r / 2$ la vitesse tangentielle moyenne dans la couche d'Ekman et sur l'épaisseur de cette couche $\delta_{\mathrm{E}}$. Ainsi, le coefficient de frottement est donné par :

$$
C_{\mathrm{f}} \propto\left(\frac{\frac{(K+1) \Omega r}{2} \delta_{\mathrm{E}}}{\nu}\right)^{-1 / 4}
$$

On note $\alpha$ l'angle formé par la tension de cisaillement à la paroi $\tau$ et la direction tangentielle. La composante tangentielle de $\tau$ s'exprime par :

$$
\tau_{\theta}=\tau \cos \alpha \propto \rho\left(\frac{K+1}{2} \Omega r\right)^{7 / 4}\left(\frac{\nu}{\delta_{\mathrm{E}}}\right)^{1 / 4}
$$

La composante radiale de la tension de cisaillement est obtenue en équilibrant la force centrifuge et la tension de cisaillement dans un élément de volume de hauteur $\delta_{\mathrm{E}}$ :

$$
\tau_{r}=\tau \sin \alpha=\rho \Omega^{2} r \delta_{\mathrm{E}}
$$

L'angle $\alpha$ d'inclinaison des lignes de courant étant constant le long d'un rayon [11], l'épaisseur de la couche d'Ekman est donnée par :

$$
\delta_{\mathrm{E}} \sim r\left(\frac{K+1}{2}\right)^{7 / 5}\left(\frac{\Omega r^{2}}{\nu}\right)^{-1 / 5}
$$

Dans la couche de Bödewadt le long du stator, on suppose que le frottement radial est contrôlé par le flux radial $Q_{\mathrm{B}}$. En considérant alors que la vitesse radiale dans le cour central est nulle, l'équation de continuité s'écrit :

$$
\overline{V_{\mathrm{E}}} \delta_{\mathrm{E}}=\frac{Q-Q_{\mathrm{B}}}{2 \pi r}
$$

$Q$ est le flux total imposé. $\overline{V_{\mathrm{E}}}$ est la vitesse moyenne dans la couche d'Ekman, proportionnelle au maximum de la vitesse atteinte dans cette couche, avec le coefficient de proportionnalité $\beta: \overline{V_{\mathrm{E}}}=\beta \Omega r$. En utilisant l'équation de continuité (7) et l'expression de $\delta_{\mathrm{E}}$, on obtient finalement :

$$
K=2 \times\left(a \times C q_{r}+b\right)^{5 / 7}-1
$$

avec $a$ et $b$ deux constantes déduites de l'expérience. La constante $b$ est obtenue à partir du cas sans flux $Q=0$. Le nouveau paramètre de similitude est un coefficient local de débit défini par $C_{q_{\mathrm{r}}}=Q R e_{r}^{1 / 5} /\left(2 \pi r^{3} \Omega\right)$, où $R e_{r}=$ $\Omega r^{2} / \nu$ est le nombre de Reynolds local. Il est à noter que, pour $r=R_{2}$, l'expression de $C_{q_{\mathrm{r}}}$ est la même que celle définie par Kurokawa et Toyokura [6].

De la loi (8), on peut déduire une loi pour le taux de cisaillement tangentiel $\tau_{\theta}$ en fonction de $C_{q_{\mathrm{r}}}$ :

$$
\tau_{\theta}=0,88 \tau_{\theta 0}\left(9,37 C q_{\mathrm{r}}+1\right)^{7 / 20}
$$

où $\tau_{\theta 0}$ est le frottement tangentiel obtenu pour $C_{q_{\mathrm{r}}}=0$. Le frottement tangentiel dans la couche d'Ekman augmente de $34 \%$ entre le cas sans flux $\left(C_{q_{\mathrm{r}}}=0\right)$ et la valeur maximale de $C_{q_{\mathrm{r}}}$ obtenue ici $\left(C_{q_{\mathrm{r}}} \simeq 0,25\right)$.

La figure 4 regroupe des centaines de mesures de vitesse et de pression pour une large gamme du nombre de Reynolds $R e=\left[6,92 \times 10^{5} ; 4,15 \times 10^{6}\right]$, et du coefficient de débit $C_{\mathrm{w}}=[-26000 ; 31000]$. On vérifie que le coefficient $K$ est indépendant du rapport d'aspect de la cavité pour $0,012 \leq G$, tant que l'hypothèse d'écoulements à couches limites séparées est vérifiée. Tous les résultats expérimentaux sont en excellent accord avec la loi analytique (8) avec $a=5,9$ et $b=0,63$. Les prévisions du modèle RSM (Reynolds Stress Modeling) de Poncet et al. [8] montrent également que $K$ dépend de $C_{q_{\mathrm{r}}}$ selon cette même loi de puissance mais avec un coefficient $a=5,3$ légèrement sous-estimé. Ceci est attribué au fait que, dans l'expérience, le niveau de prérotation varie faiblement avec le flux, alors qu'il est fixe dans le modèle. 


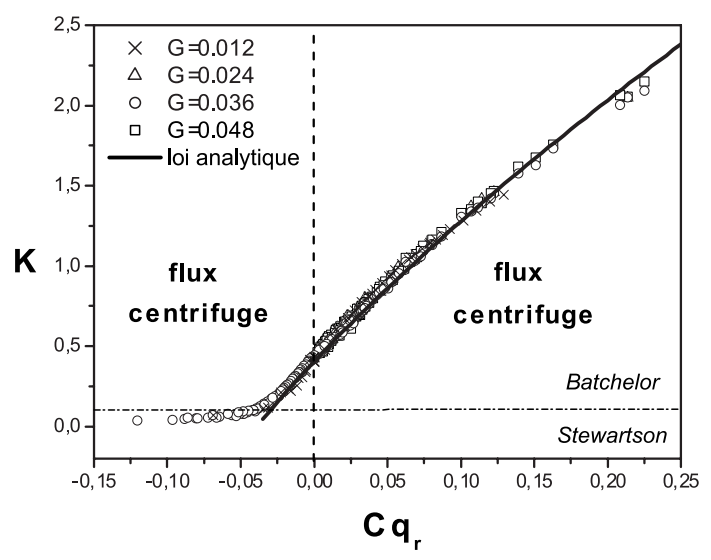

Fig. 4. Loi liant $K$ à $C_{q_{\mathrm{r}}}$ pour des écoulements turbulents de type Batchelor. Influence du rapport d'aspect de la cavité : $(\times) G=0,012,(\triangle) G=0,024,(\circ) G=0,036,(\square) G=0,048$, (-) loi analytique.

En cavité fermée $\left(C_{q_{\mathrm{r}}}=C_{\mathrm{w}}=0\right)$, la valeur de $K$ est constante 0,438 quel que soit le rayon. Pour des fortes valeurs de $C_{q_{\mathrm{r}}}$ (rayons proches de l'axe, faibles taux de rotation), la dépendance de la puissance $5 / 7$ est dominante, ce qui peut s'expliquer par le phénomène d'étirement tourbillonaire : le noyau tourne plus vite que le disque tournant $(K>1)$. Pour de faibles flux centrifuges (faibles valeurs négatives de $C_{q_{\mathrm{r}}}$ ), l'écoulement est toujours de type Batchelor et la loi analytique est encore vérifiée. Pour de plus fortes valeurs négatives de $C_{q_{\mathrm{r}}}$, la symétrie de l'écoulement de Batchelor se brise et l'écoulement est alors de type Stewartson avec une unique couche limite sur le rotor. L'hypothèse d'écoulements à couches limites séparées n'est plus vérifiée et la loi de variation de $K$ change. Le coefficient $K$ tend vers une valeur asymptotique proche de 0 . On peut noter que la transition entre ces deux types d'écoulement s'opère vers $C_{q_{\mathrm{r}}} \simeq-0,025$, de manière continue et est indépendante du rapport d'aspect (voir [8]).

\section{5 Étude paramétrique du coefficient d'entraînement $K$}

\subsection{Influence de la prérotation}

Pour les écoulements turbulents de type Batchelor en cavité fermée, le coefficient d'entraînement $K$ du fluide vaut 0,438 . Cette valeur est très supérieure à celle obtenue par Marchand [16] $K=0,313$ pour $0,3<r^{*}<0,5$ en configuration dite « infinie ». Cette différence est due à la fois au large espace radial présent entre le rotor et le stator (100 mm) mais aussi à l'absence de dispositif de prérotation dans son expérience.

Pour quantifier l'influence de la prérotation sur la loi (8), nos mesures sont comparées à celles de Debuchy [17], qui a étudié un écoulement d'air centripète à entrée radiale et sortie axiale dans une cavité rotorstator ne possédant pas de cylindre extérieur. On rappelle

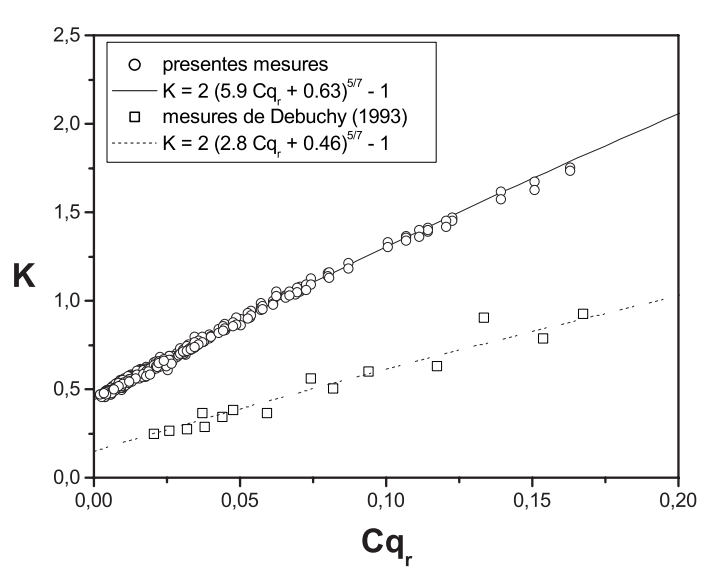

Fig. 5. Influence de la prérotation sur la loi de puissance en $5 / 7$ liant $K$ à $C_{q_{\mathrm{r}}}$.

que dans notre configuration, l'écoulement est confiné radialement et qu'une couronne assure une prérotation du fluide à l'entrée de la cavité avec $0,5 \leq K_{\mathrm{v}} \leq 0,6$ selon l'intensité du flux centripète. Les données de Debuchy [17] suivent également une loi de puissance en $5 / 7$ (Fig. 5) avec cependant deux constantes expérimentales plus faibles $(a=2,8, b=0,46)$. Cette différence est due à l'absence de prérotation du fluide et de confinement dans sa configuration. La seule influence de la mise en prérotation du fluide sur la loi d'évolution de $K$ est plus clairement mise en évidence dans le cas des écoulements laminaires (voir Sect. 5.5).

\subsection{Influence de la géométrie}

Les figures $6 \mathrm{a}$ et $6 \mathrm{~b}$ présentent l'influence de chicanes en périphérie de la cavité sur le coefficient $K$ pour des écoulements turbulents $\left(R e=4,15 \times 10^{6}\right)$ à couches limites séparées $(G=0,036)$ soumis à un flux centripète. Quel que soit le type de recouvrement $\delta$ positif ou négatif (Fig. 1b) et la hauteur entre le rotor et l'épaulement $h_{i}$, les valeurs de $K$ se distribuent sur la même loi analytique (8) que dans la cavité rotor-stator sans ajutage avec toujours $a=5,9$ et $b=0,63$. Ces chicanes engendrent des pertes de charge axiales mais pas de pertes de charge radiales et donc pas de modification du coefficient d'entraînement $K$ du fluide. La loi $K=2 \times\left(5,9 \times C_{q_{\mathrm{r}}}+0,63\right)^{5 / 7}-1$ est donc robuste aux changements de géométrie en périphérie de la cavité. Ce résultat est en accord avec les mesures de Daily et al. [7].

\section{3 Écoulements turbulents entre un disque tournant rugueux et un disque fixe lisse}

On rappelle que la loi analytique (8) est déterminée à partir du coefficient de frottement dans la couche limite d'Ekman. Kurokawa et al. [12] ont montré que la meilleure configuration pour augmenter le taux de rotation du fluide est celle où le stator est lisse $\left(k_{\mathrm{s}}^{*}=k_{\mathrm{s}} / R_{2}=3,7 \times 10^{-6}\right.$ avec $k_{\mathrm{s}}$ la hauteur moyenne des rugosités) et le rotor 


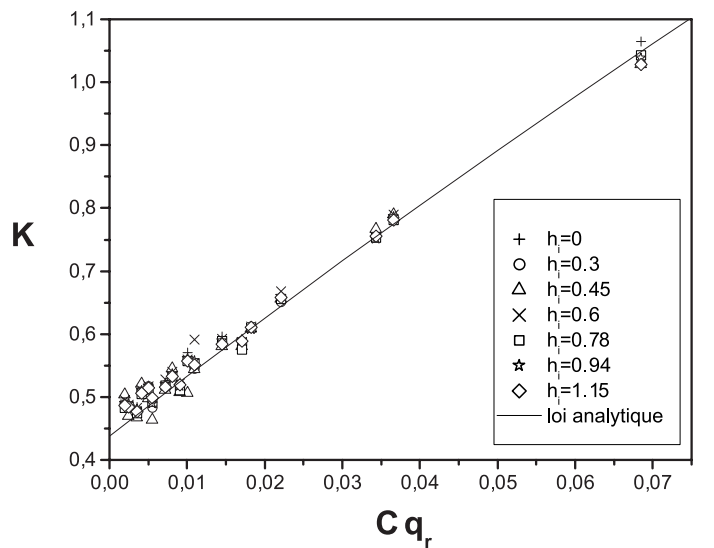

(a)

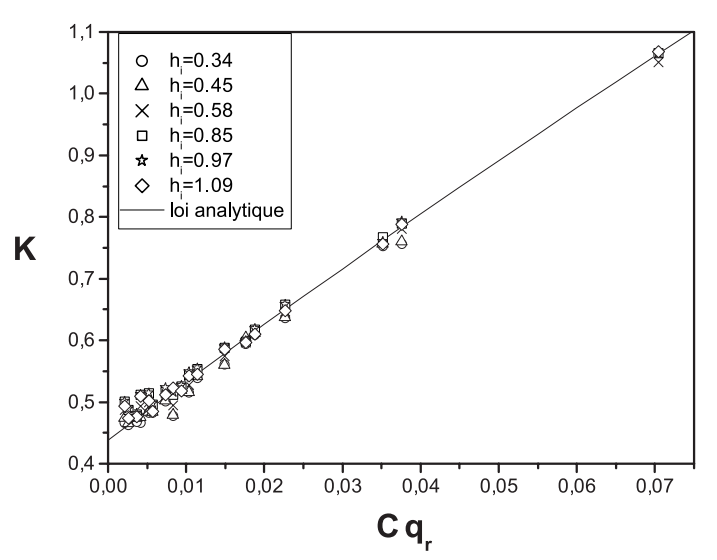

(b)

Fig. 6. Influence de la géométrie en entrée de la cavité sur la loi analytique (8) pour $G=0,036, R e=4,15 \times 10^{6}$ et $C_{\mathrm{w}}=[2579 ; 5159 ; 10317]$ (mesures par LDA) : (a) recouvrement négatif $\delta=-0,3 \mathrm{~mm}$, (b) recouvrement positif $\delta=2$ $\mathrm{mm}$.

rugueux $\left(k_{\mathrm{s}}^{*}=1,7 \times 10^{-4}\right)$. Du papier abrasif a donc été collé sur le disque tournant pour modifier le coefficient de frottement dans la couche d'Ekman. La hauteur moyenne des rugosités est supposée homogène et vaut environ $k_{\mathrm{s}}^{*}=1,2 \times 10^{-3}$. Le stator est lisse et on considère uniquement les écoulements turbulents à couches limites séparées. On peut alors appliquer la même démarche que dans le cas avec rotor et stator lisses. La « harpe » de Nikuradze [18] donne, pour cette valeur de $k_{\mathrm{s}}^{*}$, un coefficient de frottement $C_{\mathrm{f}}$ constant pour $R e \geq 10^{4}$ dans le cas de conduites rugueuses. On en déduit par la même analyse que précédemment, un coefficient de débit $C_{q_{\mathrm{rug}}}$, défini par $C_{q_{\mathrm{rug}}}=Q /\left(2 \pi \Omega r^{3}\right)$. La loi analytique permettant de déterminer $K$ s'exprime alors de la manière suivante :

$$
K=2 \times\left(a \times C_{q_{\mathrm{rug}}}+b\right)^{1 / 2}-1
$$

La figure 7 compare les mesures par LDA et la loi (10), avec les deux constantes $a=50$ et $b=0,6$. Jusqu'à $K \simeq 1,1$, la loi (10) est validée et au-delà, elle surestime les valeurs de $K$ mesurées. Les rugosités bloquent la rotation du noyau pratiquement à une vitesse de rotation

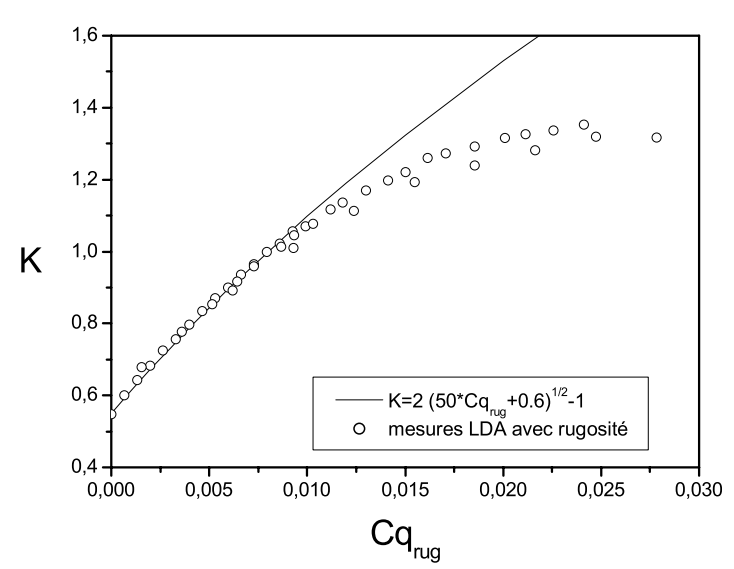

Fig. 7. Loi d'évolution de $K$ en fonction de $C_{q_{\text {rug }}}$ dans la configuration rotor rugueux/stator lisse.

qui ne dépasse pas de $30 \%$ celle du rotor. En cavité fermée $\left(C_{q_{\mathrm{r}}}=0\right), K$ augmente de $25 \%$ en présence des rugosités, ce qui confirme les résultats de Kurokawa et al. [19] pour $k_{\mathrm{s}}^{*}=1,7 \times 10^{-4}$. Cela montre, de plus, qu'au-delà d'une certaine valeur de $k_{\mathrm{s}}^{*}$, l'influence de la rugosité est faible. Jusqu'à environ $K \simeq 1,1$, le noyau tourne plus vite dans la configuration rotor rugueuxstator lisse. Au-delà de cette valeur, $K$ est, par contre, plus faible que dans la configuration rotor et stator lisses. En effet, les rugosités imposent au fluide de tourner à la vitesse du disque sur une épaisseur plus importante que dans le cas hydrauliquement lisse et contrôlent ainsi la rotation du noyau, en le ralentissant.

\section{4 Écoulements turbulents entre un disque tournant aileté et un disque fixe lisse}

Le rotor est maintenant équipé de 10 ailettes de dimensions $100 \times 10 \times 4,5 \mathrm{~mm}^{3}$ disposées en périphérie de la cavité le long d'un rayon. La valeur de l'espace interdisque $h$ est fixée à deux fois la hauteur des ailettes : $h=9 \mathrm{~mm}(G=0,036)$.

La figure 8 présente l'influence de 10 ailettes (hauteur $h / 2)$ sur le coefficient d'entraînement du fluide $K$ en $r^{*}=0,8$ (milieu des ailettes). $K$ est ici mesuré au milieu de l'espace entre le haut des ailettes et le stator. L'effet des ailettes est nettement marqué. En effet, si dans le cas du rotor lisse, ces valeurs évoluent notablement avec le coefficient de débit $C_{\mathrm{w}}$ (particulièrement pour $R e=5 \times 10^{5}$ ), les valeurs de $K$ évoluent relativement peu en présence d'ailettes et tendent quel que soit le nombre de Reynolds vers une valeur proche de 1 pour de forts flux centripètes. Pour de forts flux centrifuges, les valeurs de $K$ restent significatives contrairement au cas rotor lisse et l'écoulement préserve sa structure à couches limites séparées. L'effet des ailettes est donc de limiter la gamme de variation de $K$ suivant les valeurs de $R e$ et $C_{\mathrm{w}}$. Leur présence empêche aussi la transition entre les structurations de type Batchelor et Stewartson.

Il est à noter que les mêmes mesures ont été effectuées pour $h=6,3 \mathrm{~mm}(G=0,025)$ et qu'elles donnent lieu aux 


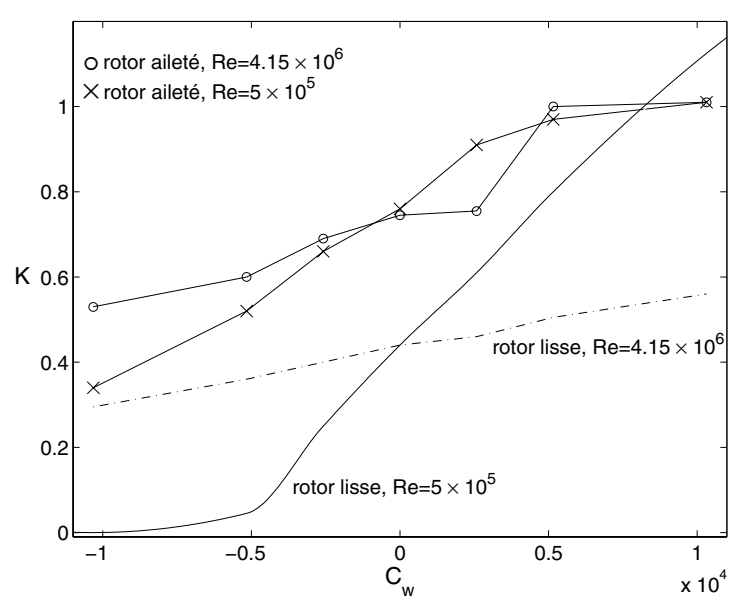

Fig. 8. Évolution de $K$ en fonction de $C_{\mathrm{w}}$ en $r^{*}=0,8$. Comparaison entre le cas lisse et le cas où le rotor est muni de 10 ailettes pour $G=0,036$.

mêmes conclusions. L'espace entre le haut des ailettes et le stator étant plus petit, le fluide situé au-dessus des ailettes est mieux entraîné que pour $h=9 \mathrm{~mm}$ et donc $K$ augmente. Par contre, aucun effet sur $K$ n'a été obtenu en faisant varier le nombre d'ailettes entre 5 et 10 .

\subsection{Extension aux écoulements laminaires entre deux disques lisses}

On considère maintenant les écoulements laminaires de type Batchelor dans une cavité rotor-stator où les deux disques sont lisses. Le coefficient de frottement vaut alors : $C_{f_{\text {moy }}}=1,328 \times R e_{\text {moy }}^{-1 / 2}[1]$. On obtient ainsi un coefficient de débit différent de celui du cas turbulent : $C_{q_{\text {lam }}}=Q \operatorname{Re}_{r}^{1 / 3} /\left(2 \pi r^{10 / 3} \Omega\right)$. La loi reliant $K$ à ce nouveau paramètre de similitude s'exprime de la manière suivante :

$$
K=a \times C_{q_{\text {lam }}}+b
$$

La dépendance linéaire de $K$ en ce coefficient de débit $C_{q_{\mathrm{lam}}}$ a été vérifiée expérimentalement (Fig. 9). Ici $a=$ 0,88 et $b=0,59$ dans le cas avec prérotation.

La loi (11) a été testée dans une seconde cavité rotor-stator possédant les mêmes caractéristiques géométriques (cylindre central en rotation, cylindre extérieur fixe ... voir [11]) mais n'ayant pas de dispositif de mise en prérotation du fluide. Les données expérimentales suivent alors la loi (11) avec $a=0,88$ et $b=0,41$. Par rapport au cas avec prérotation, le coefficient $a$ reste donc inchangé et $b$ diminue. Le taux de prérotation fixe ainsi la valeur asymptotique de $K$ dans le cas sans flux (pour $C_{q_{\text {lam }}}=0$ ). Ceci confirme les résultats obtenus pour les écoulements turbulents et montre plus clairement l'influence de la prérotation sur la loi d'évolution de $K$.

Il est à noter qu'une grande dispersion des valeurs de $K$ est obtenue pour $C_{q_{\text {lam }}}=0$. En effet, pour un écoulement laminaire en cavité fermée, $K$ varie comme l'inverse du carré du rayon [12].

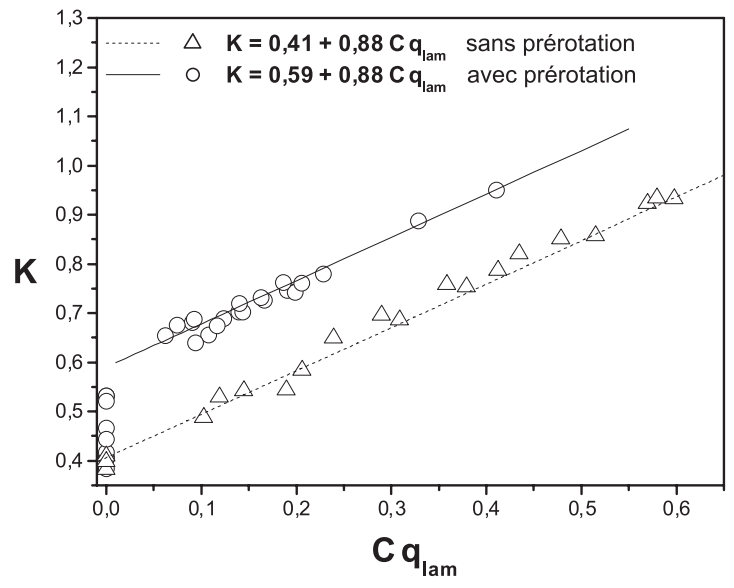

Fig. 9. Loi liant $K$ à $C_{q_{\text {lam }}}$ dans le cas d'écoulements laminaires entre deux disques lisses avec ou sans prérotation.

\section{Conclusion}

L'écoulement confiné entre un disque tournant et un disque fixe avec flux axial imposé est un écoulement représentatif de ceux rencontrés dans les turbomachines. En dehors des fines couches limites qui se développent sur chaque disque, l'écoulement est en rotation quasi solide. Cette zone est caractérisée par des vitesses radiale et axiale quasiment nulles et une vitesse tangentielle constante, qui, sous forme adimensionnée, est appelée le coefficient d'entraînement $K$ du fluide. Connaître le comportement de ce coefficient est primordial, car il est directement lié au gradient de pression radial et donc aux efforts axiaux sur le rotor.

À partir de l'expression du coefficient de frottement $C_{\mathrm{f}}$ dans la couche limite du disque tournant, nous avons déterminé des lois de puissance simples permettant de prévoir la valeur de $K$ en fonction des paramètres de l'étude : le taux de rotation $\Omega$, le flux $Q$, l'espace interdisque $h$, le taux de prérotation $K_{\mathrm{v}}$ et la position radiale $r$. Ces paramètres se regroupent sous la forme d'un coefficient local de débit $C_{\mathrm{q}}$. L'expression de ce coefficient et l'exposant des lois de puissance dépendent directement de l'expression de $C_{\mathrm{f}}$. Ces lois ont été validées expérimentalement par des centaines de mesures de vitesse et de pression pour une large gamme des paramètres de contrôle. L'influence des conditions aux limites (géométrie, niveau de prérotation, rugosité, disque aileté) a également été étudiée. Les lois ne dépendent pas de l'espace interdisque dans la limite de couches limites séparées. Elles sont également robustes à la présence de chicanes en périphérie de la cavité. Les valeurs des deux constantes contenues dans les lois analytiques sont fixées par le niveau de prérotation et par le confinement radial.

Remerciements. Les auteurs tiennent à remercier le groupe Large Liquid Propulsion de SNECMA Moteurs (Vernon, France), qui a financé le banc expérimental à travers les contrats 2003.021.G, 2004.093.G et 2005.060.G. 


\section{Références}

[1] H. Schlichting, Boundary-layer theory, 7th edition, McGraw-Hill Book Company, New-York, 1979

[2] J.W. Daily, R.E. Nece, Chamber dimension effects on induced flow and frictional resistance of enclosed rotating disks, ASME J. Basic Eng. 82 (1960) 217-232

[3] M. Itoh, Y. Yamada, S. Imao, M. Gonda, Experiments on turbulent flow due to an enclosed rotating disk, Engineering turbulence modeling and experiments, ed. W. Rodi, E.N. Ganic, New-York, 1990, pp. 659-668

[4] S.C. Cheah, H. Iacovides, D.C. Jackson, H. Ji, B.E. Launder, Experimental investigation of enclosed rotorsator disk flows, Exp. Thermal Fluid Sci. 9 (1994) 445455

[5] H. Jimbo, Investigation of the interaction of windage and leakage phenomena in a centrifugal compressor, ASME Paper, 56-A-47 1956

[6] J. Kurokawa, T. Toyokura, Study on axial thrust of radial flow turbomachinery, 2nd International JSME Symposium, Tokyo, 1972

[7] J.W. Daily, W.D. Ernst, V.V. Asbedian, Enclosed rotating disks with superposed throughflow, Report 64, M.I.T, department of civil engineering, 1964

[8] S. Poncet, M.P. Chauve, R. Schiestel, Batchelor versus stewartson flow structures in a rotor-stator cavity with throughflow, Phys. Fluids 17 (2005) 1-15

[9] L. Elena, R. Schiestel, Turbulence modeling of rotating confined flows, Int. J. Heat Fluid Flow 17 (1996) 283-289
[10] J.M. Owen, R.H. Rogers, Flow and Heat Transfer in Rotating-Disc Systems, vol. 1 Rotor-Stator Systems, ed. Morris, W.D. John Wiley and Sons Inc., New-York, 1989

[11] S. Poncet, Écoulements de type rotor-stator soumis à un flux axial : de Batchelor à Stewartson, Thèse de doctorat de l'Université d'Aix-Marseille I, 2005

[12] R.M. Gassiat, Étude expérimentale d'écoulements centripètes avec prérotation d'un fluide confiné entre un disque tournant et un carter fixe, Thèse de doctorat de l'Université d'Aix-Marseille II, 2000

[13] S. Poncet, R. Schiestel, M.P. Chauve, Centrifugal flow in a rotor-stator cavity, J. Fluid Eng. 127 (2005) 687-694

[14] R. Debuchy, A. Dyment, H. Muhe, P. Micheau, Radial inflow between a rotating and a stationary disc, Eur. J. Mech. B/Fluids 17 (1998) 791-810

[15] S. Poncet, M.P. Chauve, P. Le Gal, Turbulent rotating disk flow with inward throughflow, J. Fluid Mech. 522 (2005) 253-262

[16] O. Marchand, Sur l'écoulement entre deux disques, Thèse de doctorat de l'Université d'Aix-Marseille II, 1986

[17] R. Debuchy, Écoulement turbulent avec aspiration radiale entre un disque fixe et un disque tournant, Thèse de doctorat de l'Université des Sciences et Technologies de Lille, 1993

[18] E.A. Brun, A. Martinot-Lagarde, J. Mathieu, Mécanique des fluides 2, Dunod, Paris, 1968

[19] J. Kurokawa, T. Toyokura, M. Shinjo, K. Matsuo, Roughness effects on the flow along an enclosed rotating disk, Bull. JSME 21 (1978) 1725-1732 